\title{
Prolyl Hydroxylase Domain-2 Protein Regulates Lipopolysaccharide-Induced Vascular Inflammation
}

\author{
Qiying Fan, Hua Mao, Liang Xie, and Xinchun Pi
}

From the Cardiovascular Research Institute, Section of Athero and Lipo, Department of Medicine, Baylor College of Medicine, Houston, Texas

\author{
Accepted for publication \\ September 13, 2018. \\ Address correspondence to \\ Xinchun Pi, Ph.D., or Liang \\ Xie, Ph.D., Cardiovascular \\ Research Institute and \\ Department of Medicine, \\ Baylor College of Medicine, \\ Houston, TX 77030. E-mail: \\ xpi@bcm.edu or liangx@bcm. \\ edu.
}

\begin{abstract}
Acute lung injury and its more severe form, acute respiratory distress syndrome, are life-threatening respiratory disorders. Overwhelming pulmonary inflammation and endothelium disruption are commonly observed. Endothelial cells (ECS) are well recognized as key regulators in leukocyte adhesion and migration in response to bacterial infection. Prolyl hydroxylase domain (PHD)-2 protein, a major PHD in ECs, plays a critical role in intracellular oxygen homeostasis, angiogenesis, and pulmonary hypertension. However, its role in endothelial inflammatory response is unclear. We investigated the role of PHD2 in ECs during endotoxin-induced lung inflammatory responses with EC-specific PHD2 inducible knockout mice. On lipopolysaccharide challenge, PHD2 depletion in ECs attenuates lipopolysaccharideinduced increases of lung vascular permeability, edema, and inflammatory cell infiltration. Moreover, EC-specific PHD2 inducible knockout mice exhibit improved adherens junction integrity and endothelial barrier function. Mechanistically, PHD2 knockdown induces vascular endothelial cadherin in mouse lung microvascular primary endothelial cells. Moreover, PHD2 knockdown can increase hypoxia-inducible factor/vascular endothelial protein tyrosine phosphatase signaling and reactive oxygen speciesdependent $\mathrm{p} 38$ activation, leading to the induction of vascular endothelial cadherin. Data indicate that PHD2 depletion prevents the formation of leaky vessels and edema by regulating endothelial barrier function. It provides direct in vivo evidence to suggest that PHD2 plays a pivotal role in vascular inflammation. The inhibition of endothelial PHD2 activity may be a new therapeutic strategy for acute inflammatory diseases. (Am J Pathol 2019, 189: 200-213; https://doi.org/10.1016/ j.ajpath.2018.09.012)
\end{abstract}

Acute lung injury (ALI) and its more severe form, acute respiratory distress syndrome (ARDS), are syndromes of acute respiratory failure with substantial morbidity and mortality. ${ }^{1}$ Although progress has been made in the understanding of the etiology, epidemiology, and treatment of these diseases, the mortality rate of ALI is still approximately $40 \%$ in the United States. ${ }^{2}$ Even in patients who survive ALI, their quality of life is usually severely affected. Therefore, novel therapies are needed to improve clinical outcomes. Clinical features in patients with ALI/ARDS include immune system activation, microvascular injury, and diffuse alveolar damage with intrapulmonary hemorrhage, edema, and fibrin deposition. The pathogenesis of ALI/ARDS is characterized by acute inflammatory responses to infections caused by injury to the vascular endothelium and alveolar epithelium, associated with systemic illness, such as sepsis or major trauma. ${ }^{2}$ The pulmonary endothelium, a semipermeable barrier, is not only affected by pulmonary inflammation but also plays a regulatory role during the injury. Endothelial cells (ECs) regulate pulmonary permeability by controlling the interchange of macromolecules and fluid between the blood and alveolar space in lung tissue. During severe sepsis, ECs respond to bacterial endotoxin liposaccharide (LPS) in the blood and adjust from a low to a high permeability barrier, eventually resulting in increased flux of proteins, fluid, and immune cells across vessels into tissues. ${ }^{3,4}$ They also actively

Supported by NIH R01s HL112890 and HL061656 (X.P.) and HL122736 (L.X.).

Disclosures: None declared. 
produce and secrete proinflammatory cytokines to further activate immune cells, such as neutrophils, monocytes, and tissue macrophages. ${ }^{5}$ However, the underlying mechanisms mediating endothelial activation in response to severe sepsis or other predisposing clinical factors are still not fully understood.

In the maintenance of endothelial permeability, the dynamic opening of interendothelial junctions, including tight junctions, adherens junctions (AJs), and gap junctions, controls the passage of small molecules and inflammatory cells through the paracellular pathway. ${ }^{6}$ AJs play a dominant role in endothelial barrier function, ${ }^{7}$ whereas tight junctions regulate the tightness of the endothelial barrier and neutrophil transmigration $^{8,9}$ and gap junctions mainly facilitate cell-cell communication via the phosphorylation of connexins. ${ }^{10} \mathrm{AJs}$ are formed by the hemophilic cis and trans dimers of vascular endothelial cadherin (VE-cadherin; alias cadherin-5) between adjacent endothelial cells. Inhibition of VE-cadherin activity by its neutralizing antibody or mutants results in disruption of AJs and increase in endothelial permeability. ${ }^{6}$ AJs disassembly is controlled by VE-cadherin endocytosis, which is regulated via p120 catenin phosphorylation by protein kinase $\mathrm{C} \alpha$ or VE-cadherin phosphorylation by Src induced by inflammatory mediators. ${ }^{11,12}$ On the other hand, vascular endothelial protein tyrosine phosphatase (VE-PTP), a transmembrane phosphatase, can stabilize the VE-cadherin protein level via dephosphorylating VE-cadherin. ${ }^{13}$ The interaction of VE-PTP and VE-cadherin can be disrupted by LPS or vascular endothelial growth factor (VEGF), contributing to vascular leakage. ${ }^{14}$ In addition, VE-cadherin expression is regulated by transcription factors, such as ETS-related gene, erythroblast transformation-specific transcriptional factor-1, and T-cell acute lymphoblastic leukemia-1/stem cell leukemia. ${ }^{15-17}$ However, signaling pathways regulating these transcriptional events on inflammatory stimulation remain largely unknown.

Although hypoxia can induce inflammatory responses, inflammation itself is also often a main cause for tissue hypoxia. Oxygen sensing and hypoxia signaling pathways are critical in inflammatory diseases. ${ }^{18}$ Hypoxia-inducible factor (HIF) is a key transcription factor governing a large set of gene expression in an oxygen-dependent manner. Infection with pathogen can stabilize HIFs and induce HIF-dependent gene expression. ${ }^{18}$ Recent studies demonstrate that HIF2 $\alpha$ can protect AJ integrity via up-regulating VE-PTP expression and decreasing VE-cadherin phosphorylation. ${ }^{15,19}$ Prolyl hydroxylases (PHDs 1, 2, and 3) play essential roles in the oxygen-sensing system through hydroxylating $\mathrm{HIF} \alpha$ and targeting it to proteasome for degradation. ${ }^{20-22}$ Hypoxia inhibits the activity of PHDs, resulting in stabilization of HIFs and activation of their transcriptional activity. ${ }^{23}$ PHD enzymes exhibit specific and nonredundant in vivo functions. PHD1 plays a key role in mitochondrial energy metabolism of liver and skeletal muscle. ${ }^{24,25}$ PHD2 and PHD3 perform their specific functions in the development of the heart and sympathoadrenal system, respectively. ${ }^{26,27}$ Reports also show that
PHD2 and PHD3 can act cooperatively in pathophysiologic conditions, such as hepatic steatosis and dilated cardiomyopathy. ${ }^{28,29}$ Systemic depletion of PHD3 $\left(\mathrm{PHD}^{-/-}\right.$) mice, but not $\mathrm{PHD}^{-/-}$or PHD2 ${ }^{+/-}$, aggravates death events, resulting from LPS-induced sepsis due to hyperactivated innate immune cells lacking PHD3. ${ }^{30}$ On the contrary, mice with PHD1 depletion are less susceptible to dextran sulfate sodium-induced colitis. ${ }^{31}$ Dimethyloxalylglycine (DMOG), a pan inhibitor of PHDs, and RNA interference of PHD1 or PHD2 can attenuate LPS-induced tumor necrosis factor (TNF) $-\alpha$ expression in macrophages, which is independent of HIF1 $\alpha$ signaling. ${ }^{32}$ These data indicate that each PHD enzyme may possess distinctive and even opposite roles in the inflammatory responses. However, the molecular mechanisms under their different functional roles in inflammation remain unclear.

Because endothelial cells play a pivotal role in LPSinduced inflammatory responses, we studied the role of PHDs by evaluating how endothelial cell-specific PHD2 or PHD2 and PHD3 double-inducible knockout mice respond to LPS-induced acute lung injury. In this study, it was observed that the loss of PHD2, but not PHD3, in endothelial cells protects pulmonary vascular leakage on LPS challenge. Mice with PHD2 depletion in endothelial cells display alleviated vascular inflammatory responses, showing increased wet/dry lung weight ratio, pulmonary edema, and production of proinflammatory cytokines. Endothelial AJ integrity and barrier function are improved in PHD2-depleted mice. Mechanistically, PHD2 knockdown results in the stabilization of HIF $1 \alpha / 2 \alpha$, increases of reactive oxygen species (ROS) generation, and p38 activation, thereby up-regulating the VEcadherin protein level. These findings suggest that PHD2 depletion in endothelial cells protects mice from LPS-induced pulmonary injury. Our findings suggest that PHD2 may act as a new therapeutic target against acute lung injury, sepsis, and related inflammatory diseases.

\section{Materials and Methods}

\section{Generation of EC-Specific PHD2 ${ }^{\mathrm{f} / \mathrm{f}}$ Mice and LPS Administration}

To generate $\mathrm{PHD}_{2}^{\mathrm{f} / \mathrm{f}} ; \mathrm{Cdh} 5-\mathrm{CreER}{ }^{+/-}[\mathrm{PHD} 2$ endothelialspecific knockout (eKO)], $\mathrm{PHD}^{\mathrm{f} / \mathrm{f}}$; $\mathrm{Cdh} 5-\mathrm{CreER}^{+/-}(\mathrm{PHD} 3$ $\mathrm{eKO})$, and $\mathrm{PHD} 2 / 3^{\mathrm{f} / \mathrm{f}} ; \mathrm{Cdh} 5-\mathrm{CreER}^{+/-}(\mathrm{PHD} 2 / 3 \mathrm{eKO})$ mice, PHD2 $2^{\text {flox/flox }}\left(\mathrm{PHD}^{\mathrm{f} / \mathrm{f}}\right)$ and PHD3 $3^{\text {flox/flox }}\left(\mathrm{PHD}^{\mathrm{f} / \mathrm{f}}\right)$ mice [both generously provided by Dr. Guo-Hua Fong (University of Connecticut Health Center, Farmington, CT)] were crossed with $\mathrm{Cdh} 5-\mathrm{CreER}^{+/-}$transgenic mice [generously provided by Dr. Ralf H. Adams (Max Planck Institute for Molecular Biomedicine, Münster, Germany)] and expanded on a C57BL/6 genetic background from breeders. Mice were housed in microisolator cages under pathogen-free conditions and subjected to 12-hour light/ dark cycles. PHD2, PHD3, and PHD2/3 eKO male mice and their littermate controls [wild type (WT), PHD2 $2^{\mathrm{f} / \mathrm{f}}$; Cdh5-CreER ${ }^{-l-}, \mathrm{PHD}^{\mathrm{f} / \mathrm{f}}$; $\mathrm{Cdh} 5-\mathrm{CreER}^{-/-}$, or PHD2/3/f; 
Cdh5-CreER ${ }^{-1-}$ mice], at 7 weeks of age, were injected intraperitoneally with tamoxifen $(20 \mathrm{mg} / \mathrm{kg}$ per day; Sigma, St. Louis, MO) for 3 days to induce endothelial cell-specific PHD2 depletion. After 1 week, LPS $(10 \mathrm{mg} / \mathrm{kg}$, lethal dose; $8 \mathrm{mg} / \mathrm{kg}$, sublethal dose; Sigma) was administrated via i.v. injection. In addition, C57BL/6J mice at 8 weeks of age were injected intraperitoneally with saline or a pan inhibitor of PHDs, DMOG (8 mg; Frontier Scientific, Logan, UT). Two hours later, mice were challenged with LPS (10 mg/kg, intravenously). All studies were performed according to protocols reviewed and approved by the Baylor College of Medicine (Houston, TX) Institutional Animal Care and Use Committee.

\section{Isolation of Mouse Endothelial Cells}

Isolation of mouse lung endothelial cells was performed, as previously described. ${ }^{33}$ Briefly, lung tissues were removed and placed in a petri dish containing $20 \%$ fetal bovine serum in Dulbecco's modified Eagle's medium with high glucose. Minced lung tissues were then put into $25 \mathrm{~mL}$ of prewarmed collagenase $(2 \mathrm{mg} / \mathrm{mL})$ solution at $37^{\circ} \mathrm{C}$ for 1 hour. The cell suspension was centrifuged in a plastic tube at $400 \times g$ for 8 minutes at $4^{\circ} \mathrm{C}$, and the cell pellet was resuspended in cold phosphate-buffered saline (PBS) with $0.1 \%$ bovine serum albumin. Anti-mouse platelet endothelial cell adhesion molecule 1 Dynabeads (Thermo Fisher Scientific, Waltham, MA) were then added into the cell suspension. The pulled down mouse lung endothelial cells were resuspended in endothelial growth medium (MCDB 131; $100 \mu \mathrm{g} / \mathrm{mL}$ heparin, $100 \mu \mathrm{g} / \mathrm{mL}$ endothelial cell growth supplement, $10 \mu \mathrm{g} / \mathrm{mL}$ epidermal growth factor, $2 \mathrm{mmol} / \mathrm{L} \mathrm{L}$-glutamine, $1 \mathrm{mg} / \mathrm{mL}$ hydrocortisone, and $1 \%$ antibiotics). ${ }^{34}$

\section{Lung Wet/Dry Weight Ratio}

The proportion of extravascular lung water was calculated as an index of lung edema. The whole lung was excised and weighed. The whole lung was then dried at $60^{\circ} \mathrm{C}$ in a heat block until a contact weight was obtained, and the wet/dry weight ratio was calculated.

\section{Evans Blue Staining}

Evans Blue Dye (EBD; 1\%; Sigma) was dissolved in PBS, and $200 \mu \mathrm{L}$ was injected intravenously into the mice 16 hours after LPS administration. After 30 minutes, mice were euthanized. Intravascular EBD was washed out by a $5-\mathrm{mL}$ PBS perfusion. The whole lung was excised and then homogenized in $1 \mathrm{~mL}$ of formamide, then incubated at $60^{\circ} \mathrm{C}$ for 24 hours, followed by centrifugation at $5000 \times g$ for 30 minutes. Supernatants were collected and measured at 620 and $740 \mathrm{~nm}$ using a Tecan Infinite 200 Pro microplate reader (Tecan, Morrisville, NC). The EBD concentration was determined from standard absorbance curves that were measured in parallel. The correction for the heme-containing pigments is calculated by the following formula: $\mathrm{EBD}=\mathrm{E}_{620}-\left(1.426 \times \mathrm{E}_{740}+0.030\right)$.

\section{BALF Collection, Cell Count, and Protein Concentration}

Mouse lungs were lavaged with $800 \mu \mathrm{L}$ PBS. The retained bronchoalveolar lavage fluid (BALF) was centrifuged at $500 \times$ $g$ for 5 minutes at $4^{\circ} \mathrm{C}$. Supernatants were collected as BALF and stored at $-80^{\circ} \mathrm{C}$ until assessed for cytokine concentration, and cell pellets were resuspended in $200 \mu \mathrm{L}$ ACK lysis buffer (Quality Biological, Gaithersburg, MD). Total cell numbers were counted using a hemocytometer. The protein concentration was measured by bicinchoninic acid assay.

\section{Measurement of Cytokines in BALF and Serum}

The concentration of cytokines was quantified via the analysis of BALF or serum with enzyme-linked immunosorbent assay kits for IL-6 and TNF- $\alpha$ (R\&D Systems, Minneapolis, $\mathrm{MN}$ ), according to the manufacturer's instructions.

\section{MPO Activity}

Myeloperoxidase (MPO) activity in BALF was assessed using an MPO peroxidation fluorometric assay kit (Cayman Chemical, Ann Arbor, MI), according to the manufacturer's instructions.

\section{Transfection of Mouse Lung MLECs with siRNAs}

The stealth siRNA duplexes designed to suppress the expression of PHD2 (5'-AUACAUGUCACGCAUCUUCCAUCUC-3') were purchased from Life Technologies (Carlsbad, CA). SB203580 was purchased from Sigma. p38 siRNA was purchased from Cell Signaling Co (number 6417; Danvers, MA). siRNAs were transfected into microvascular primary ECs (MLECs), according to a previously published protocol. ${ }^{35}$ Briefly, MLECs (Cell Biologics, Chicago, IL) were transfected with $100 \mathrm{pmol}$ siRNAs. Indicated treatments and cell harvesting with siRNA-transfected MLECs were performed 2 days later.

\section{Permeability Assay}

Permeability assay was measured by the passage of fluorescein isothiocyanate (FITC)-conjugated dextran (10 $\mathrm{kDa}$ ), as previously described. ${ }^{36}$ Briefly, MLECs were transfected with indicated siRNAs. One day later, cells were plated onto transwell collagen-coated membrane inserts for 2 days to form a monolayer. Cells in inserts were then treated with $10 \mu \mathrm{g} / \mathrm{mL}$ LPS for 24 hours. The receiver plate contains $500 \mu \mathrm{L}$ of the same growth medium. FITC-dextran $(10 \mathrm{kDa})$ at 1:40 dilution was added into the inserts for 1 hour. The medium in the receiver wells was then removed to a black 96-well plate for fluorescence measurement at 485-nm excitation/535-nm emission wavelengths by using a Tecan Infinite M200 Pro microplate reader. 
Lung Histology and Immunofluorescence

Mouse lungs were harvested 24 hours after the administration of LPS and fixed in 4\% paraformaldehyde for 24 hours. Tissues were embedded in paraffin. Sections $(5 \mu \mathrm{m}$ thick) were stained with hematoxylin and eosin and observed by light microscopy. Lung injury was graded from 0 (normal) to 3 (severe) in four categories: interstitial inflammation, neutrophil infiltration, congestion, and edema, following previous reports. ${ }^{37}$ The injury score was calculated by adding the individual scores for each category. Scoring was performed blindly (X.P.). Lung tissues were also prepared for frozen sectioning. Frozen sections were used for immunofluorescence imaging for VE-cadherin expression. Immunofluorescence imaging with cultured ECs was performed, as previously described. ${ }^{35}$ The relative intensity of nuclear factor erythroid-2-related factor 2 (NRF2) in nuclear and cytoplasmic fractions of each cell was quantified with ImageJ software version 1.51j8 (NIH, Bethesda, MD; http://imagej.nih.gov/ij).

\section{ROS Generation}

ROS were detected by staining MLECs with CM$\mathrm{H}_{2}$ DCFDA (the chloromethyl derivative of $2^{\prime}, 7^{\prime}$-dichlorodihydrofluorescein diacetate) or dihydroethidium (DHE; Thermo Fisher Scientific). CM- $\mathrm{H}_{2}$ DCFDA is oxidized to green fluorescent DCF by hydrogen peroxide, and DHE, as a superoxide indicator, is oxidized to red fluorescent ethidium. Cells were loaded with $5 \mu \mathrm{mol} / \mathrm{L}$ CM- $\mathrm{H}_{2}$ DCFDA or $10 \mu \mathrm{mol} / \mathrm{L}$ DHE for 30 minutes, followed by LPS treatment for 2 hours. ROS were blocked by antioxidant $\mathrm{N}$-acetyl-L-cysteine (NAC) or NADPH oxidase inhibitor diphenyleneiodonium (DPI), which were purchased from Sigma. After a PBS wash, cell lysates were collected and the mean fluorescence intensity was determined as ROS generation by a Tecan microplate reader (for CM- $\mathrm{H}_{2}$ DCFDA: excitation, $485 \mathrm{~nm}$; and emission, $530 \mathrm{~nm}$; for DHE: excitation, $520 \mathrm{~nm}$; and emission, $620 \mathrm{~nm}$ ).

\section{Terminal Deoxynucleotidyl Transferase-Mediated dUTP} Nick-End Labeling Assay

Terminal deoxynucleotidyl transferase-mediated dUTP nick-end labeling staining was performed by using the ApopTag peroxidase in situ apoptosis detection kit (S7100; Millipore, Burlington, MA), following a previously published protocol. ${ }^{38}$ Images were taken with confocal laser scanning microscopy.

\section{Real-Time PCR}

Total RNAs were reverse transcribed into cDNAs with the iScript cDNA synthesis kit (Bio-Rad, Hercules, CA). The specific primers used for the real-time PCR are the following: PHD2, 5'-GGGACTGTACTGTGGGGTCA-3' (forward) and $5^{\prime}$-TCCGACAGCGTCTCCTCT-3' (reverse); PHD3, 5'CAGGTTATGTTCGCCATGTG- $3^{\prime}$ (forward) and $5^{\prime}$ AGGACCCCTCCGTGTAACTT-3' (reverse); VE-cadherin, $5^{\prime}$-GTTCAAGTTTGCCCTGAAGAA-3' (forward) and $5^{\prime}$ GTGATGTTGGCGGTGTTGT-3' (reverse); and VE-PTP, $5^{\prime}$-AAGCCACACACCGCCTAC-3' (forward) and $5^{\prime}$ TCAAGTCCTCGTAAATAGCTG-3' (reverse).

\section{Western Blot Analysis}

Cells were harvested in lysis buffer (1\% Triton X-100, 50 $\mathrm{mmol} / \mathrm{L}$ tris, $\mathrm{pH} 7.4,150 \mathrm{mmol} / \mathrm{L} \mathrm{NaCl}, 1 \mathrm{mmol} / \mathrm{L} \mathrm{Na}_{3} \mathrm{VO}_{4}$, and $0.1 \%$ protease inhibitor mixture; Sigma) and clarified by centrifugation at $15,000 \times g$. Proteins were separated by SDS-PAGE and transferred onto $0.45-\mu \mathrm{m}$ polyvinylidene difluoride membranes. The primary antibodies used were as follows: anti-VE-cadherin (CD144; number 555289; BD Biosciences, San Jose, CA); anti-HIF1 $\alpha$ (number 14179; Cell Signaling, Danvers, MA); anti-HIF2 $\alpha$ [number ab199 (Abcam, Cambridge, UK) and sc-13596 (Santa Cruz Biotechnology, Dallas, TX); anti-PHD2 (number 4835; Cell Signaling); anti-p-p38 (number 4511; Cell Signaling); antip38 (number 9212; Cell Signaling); and anti-NRF-2 (number 62352; Abcam). $\beta$-Actin was used as a loading control. Horseradish peroxidase-conjugated anti-mouse, anti-rabbit, and anti-rat IgG heavy and light chains (GE Healthcare, Chicago, IL) were used as the secondary antibodies.

\section{Statistical Analysis}

All values were expressed as means \pm SEM. Statistical analyses were performed using the $t$-test for two groups or by analysis of variance and followed by a post hoc test with a correction when needed for multiple groups. $P \leq 0.05$ was considered statistically significant.

\section{Results}

Endothelial Cell-Specific PHD2 Depletion Attenuates LPS-Induced Acute Lung Injury

Previous studies demonstrate that PHD3 global deficient mice are more susceptible to LPS-induced sepsis shock, whereas mice with PHD1 deficiency display no significant changes compared with their WT counterparts. ${ }^{30}$ PHD2 knockout mice are embryonic lethal, ${ }^{39}$ and mice with PHD2 haploinsufficiency demonstrate similar inflammatory responses as their littermate controls. ${ }^{30}$ To further understand the roles of endothelial PHDs in acute inflammatory responses, an endothelial cell-specific inducible knockout strategy was used to deplete PHD2 or both PHD2 and PHD3 in endothelial cells only during adulthood. Endothelial cell-specific PHD2 or PHD2/3 inducible knockout (PHD2 or $\mathrm{PHD} 2 / 3$ eKO) mice were generated by crossing $\mathrm{PHD}^{\text {flox/flox }}\left(\mathrm{PHD}^{\mathrm{f} / \mathrm{f}}\right)$ or PHD2/3 ${ }^{\mathrm{f} / \mathrm{f}}$ with $\mathrm{Cdh} 5-\mathrm{CreER}^{+/-}$ 
mice, followed by tamoxifen injection to induce Cdh5mediated endothelial-specific disruption of PHD2 or both PHD2 and PHD3. As expected, on tamoxifen injection, lung ECs isolated from PHD2 eKO mice displayed $>80 \%$ reduction of PHD2 mRNA levels compared with those from their littermate control (WT; PHD2 ${ }^{\mathrm{f} / \mathrm{f}} ; \mathrm{Cdh} 5-\mathrm{CreER}^{-1-}$ ) mice (Figure 1A). However, no obvious decreases were observed in other tissues, including heart, lung, or kidney. Reports have shown that knocking down PHD2 expression can induce the PHD3 expression, likely because of a compensative response. $^{28}$ Consistently, PHD3 mRNA levels increased in ECs isolated from the PHD2 eKO mice compared with WT ECs (data not shown). On the other hand, in the PHD2/3 eKO mice, both PHD2 and PHD3 were knocked down in endothelial cells (Supplemental Figure S1A).

Next, we determined whether the depletion of PHD2 and PHD3 in endothelial cells affects acute inflammatory responses when mice are challenged with LPS. After mice were administrated with a lethal dose $(10 \mathrm{mg} / \mathrm{kg})$ of LPS intravenously, all WT mice died within 2 days. However, PHD2 and PHD2/3 eKO mice recovered from this challenge, with a 7-day survival rate at a similar level $(50.0 \%$ and $46.2 \%$, respectively), whereas PHD3 eKO mice demonstrated a low (20\%) survival rate (Figure 1B and Supplemental Figure S1B). The PHD inhibitor, DMOG, also increased the survival rate in response to LPS challenge (Figure 1C). These results demonstrate that endothelial PHD2 is the main player during LPS-induced endotoxemia, and the inhibition of PHD activity is protective from sepsis shock. Next, lung injury-associated parameters of PHD2 eKO mice were compared with their littermate control when mice were challenged with a sublethal dose of LPS ( $8 \mathrm{mg} / \mathrm{kg}$, intravenously). Pulmonary edema, indicated by a significantly increased ratio of lung wet/dry weight, was observed in WT mice after LPS challenge compared with saline control groups (Figure 1D). However, pulmonary edema was not detected in PHD2 eKO mice at either basal conditions or in response to LPS challenge. Capillary permeability changes were also evaluated by measuring extravasated EBD content from circulation into the lung, an indicator of elevated capillary permeability. Indeed, extravasated EBD content in the lung tissue of PHD2 eKO mice was significantly less than that from WT mice after LPS treatment (Figure 1E). Last, LPS-induced histopathological changes of lung tissue were determined. Marked decreases of inflammatory cell infiltration, interalveolar septal thickening, and interstitial edema, together with a lower lung injury score, were observed in the lung of PHD2 eKO mice, compared with those of WT mice after LPS treatment (Figure 1, F and G). Taken together, PHD2 eKO mice display a significant alleviation in lung injury phenotype, indicating that PHD2 depletion in endothelial cells protects mice from LPS-induced pulmonary injury.

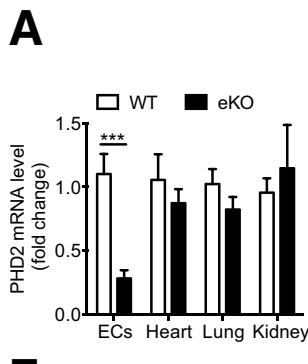

$\mathbf{F}$
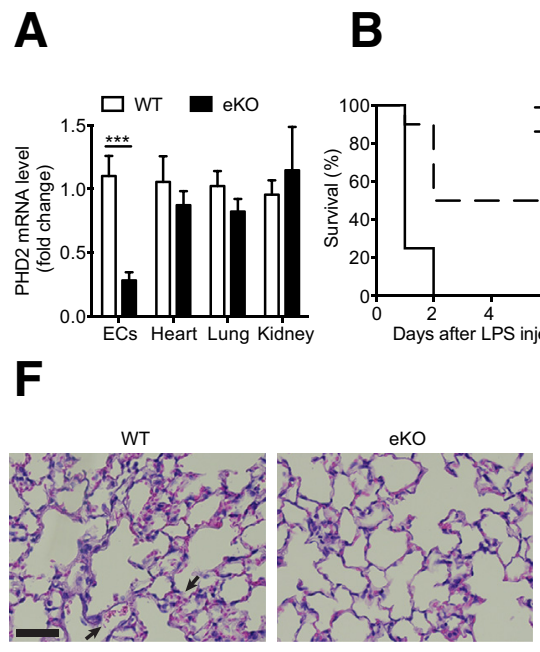
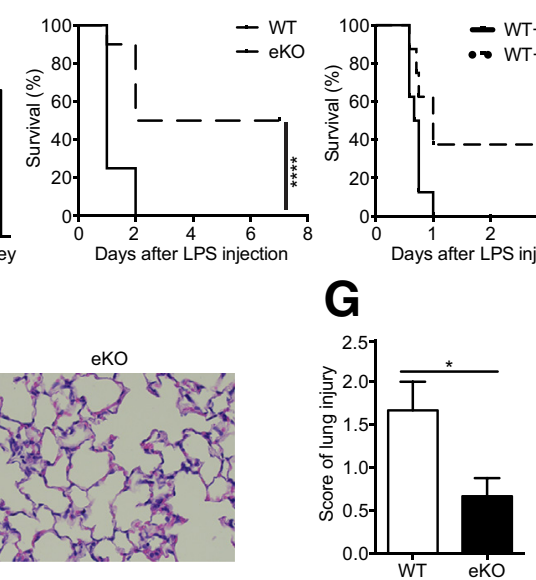

D
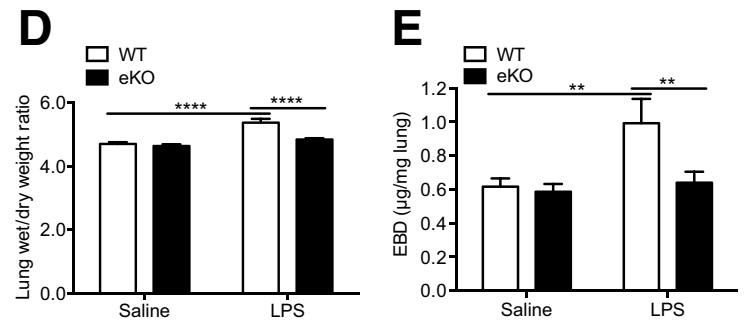

Figure 1 PHD2 depletion in endothelial cells protects lipopolysaccharide (LPS)-induced pulmonary vascular leakage and lung injury. A: PHD2 mRNA is specifically depleted in endothelial cells (ECs), but not in heart, lung, or kidney. ECs and other tissues were isolated from PHD2 eK0 or their littermate control (WT) mice. All mice were injected with tamoxifen. PHD2 mRNA levels were measured with isolated tissue RNAs via real-time quantitative PCR. B: The survival rate of PHD2 eK0 mice is higher than their littermate control on LPS challenge. PHD2 eK0 and WT mice were subjected to a lethal dose of LPS (10 mg/kg, intravenously). Their survival was monitored and compared. C: C57BL/6J mice were injected with 8 mg dimethyloxalylglycine (DMOG), a pan inhibitor of PHDs. Two hours later, mice were subjected for LPS challenge. D and E: WT and eK0 mice were challenged with a sublethal dose of LPS (8 mg/kg, intravenously) for 16 hours. Acute pulmonary responses, including increases of lung edema and permeability, were measured by lung wet/dry weight ratio (D) and Evans Blue Dye (EBD) assay (E). F: Representative photomicrographs of lung tissues stained with hematoxylin and eosin at 24 hours after LPS injection. LPS stimulated infiltration of inflammatory cells into lung interstitium and alveolar spaces (arrows), alveolar wall thickening, and intra-alveolar exudation. G: Lung tissues were screened for lung injury score. Data are expressed as means \pm SEM. $n=4$ (A, WT); $n=5(\mathbf{A}$, eK0); $n=10$ (B); $n=8(\mathbf{C}) ; n=7$ to 13 (D and E); $n=6$ (G). ${ }^{*} P<0.05,{ }^{*} P<0.01,{ }^{*} * P<0.001$, and ${ }^{* * * * P}<0.0001$ [analysis was by two-way analysis of variance, followed by Fisher's least significant difference multiple-comparison test $(\mathbf{A}, \mathbf{D}$, and $\mathbf{E})$, log-rank test $(\mathbf{B}$ and $\mathbf{C})$, and unpaired $t$-test $(\mathbf{G})]$. Scale bar $=50 \mu \mathrm{m}(\mathbf{F})$. 0 riginal magnification, $\times 200(\mathbf{F})$. 


\section{LPS-Induced Lung Inflammatory Responses Are} Attenuated in PHD2 eKO Mice

Endotoxemia-induced lung tissue damage is coupled with the inflammatory response, displaying exaggerated infiltration of immune cells and the secretion of cytokines into airspace compartments of lung tissue. Therefore, several parameters of lung inflammation were determined, including the MPO activity, cell infiltration, protein content, and accumulated cytokines in BALF. As expected, LPS challenge with WT mice enhanced MPO activity up to 3.39-fold in BALF (Figure 2A), indicating an increase in leukocyte infiltration. However, this increase was completely blocked in PHD2 eKO mice (Figure 2A). In addition, a robust increase of recovered cell number in BALF was observed in WT mice after LPS injection, compared with WT mice that received saline, indicating an increased leukocyte infiltration (Figure 2B). However, cell count in BALF of PHD2 eKO mice was significantly lower than that of WT mice. These results suggest that PHD2 depletion in endothelial cells blocks leukocyte infiltration into the alveolar space. Next, total protein content was measured in BALF, and the protein level was dramatically decreased in PHD2 eKO mice compared with WT mice in response to LPS (Figure 2C). Further analysis with enzyme-linked immunosorbent assay demonstrated that LPS challenge increased protein levels of IL- 6 and TNF- $\alpha$ in BALF and serum of the WT mice (Figure 2, D-G). But, PHD2 depletion in ECs significantly blocked the increases of these cytokine levels in BALF and the TNF- $\alpha$ level in serum (Figure 2, D-G). Taken together, these data indicate that loss of PHD2 expression in endothelial cells alleviates acute lung inflammation through blocking leukocyte infiltration and proinflammatory cytokine production.

\section{EC-Specific PHD2 Depletion Protects Endothelial Barrier Integrity through Controlling AJ Integrity and Inhibiting Endothelial Apoptosis}

Inhibition of PHD2 by its specific chemical inhibitor promotes lung adherens junction integrity and reduces mortality in LPS or cecal ligation and puncture-induced lung injury models. ${ }^{19}$ A similar protective role during LPS-induced lung injury is identified in the PHD2 eKO mice (Figures 1 and 2), suggesting that the regulation of endothelial adherens junction and vascular permeability by endothelial PHD2 activity might provide an important mechanistic explanation for the alleviated pulmonary inflammatory responses in PHD2 eKO mice. Therefore, it was investigated whether PHD2 depletion affects vascular AJ integrity and endothelial cell permeability. VE-cadherin plays an important role in maintaining normal endothelial barrier function. In response to inflammatory injury, VE-cadherin endocytosis or down-regulation of VE-cadherin expression induces disassembly of the adherens junction. ${ }^{40}$ The VE-cadherin protein level was examined in pulmonary endothelium of PHD2 eKO mice. Visualization of VE-cadherin-positive AJs showed that PHD2 depletion preserved AJ integrity, demonstrating an increase in VE-cadherin staining signals at AJs of pulmonary arterioles (Figure 3, A and B). The effects

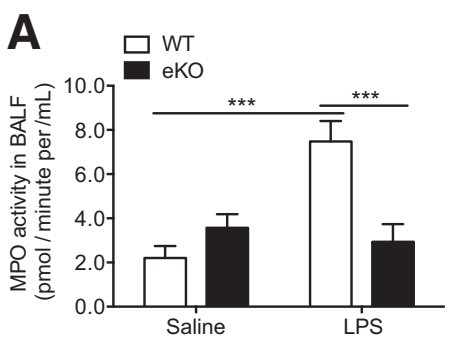

D

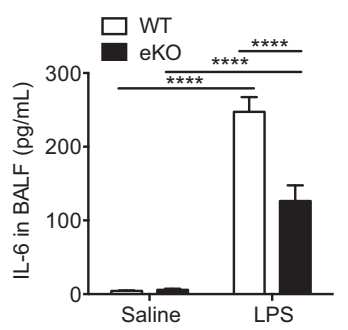

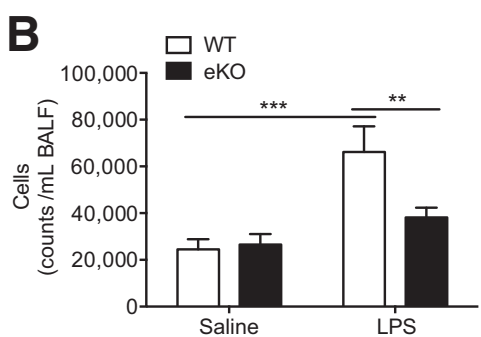

$\mathbf{F}$

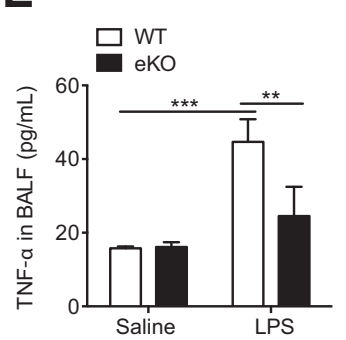

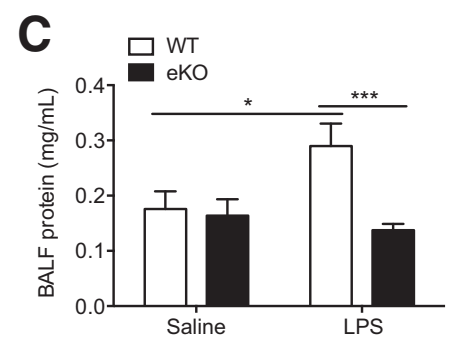

G
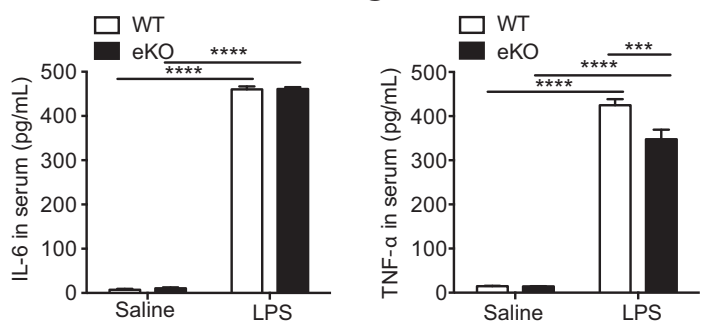

Figure 2 PHD2 depletion in endothelial cells attenuates lipopolysaccharide (LPS)-induced lung inflammatory responses. WT and PHD2 eK0 mice were treated with LPS ( $8 \mathrm{mg} / \mathrm{kg}$, intravenously) for 6 hours. LPS-induced lung inflammation was evaluated with measurements of myeloperoxidase (MP0) activity (A), total cell number (B), and total protein concentration (C) in bronchoalveolar lavage fluid (BALF). The production of proinflammatory cytokines was assessed by an enzyme-linked immunosorbent assay for levels of IL- 6 (D and $\mathbf{F}$ ) and tumor necrosis factor (TNF)- $\alpha$ (E and $\mathbf{G}$ ) in BALF and serum, respectively. Data are expressed as means \pm SEM. $n=4$ to $5(\mathbf{A}) ; n=6$ to 8 (B); $n=5$ to $8(\mathbf{C}-\mathbf{G})$. ${ }^{*} P<0.05,{ }^{*} P<0.01,{ }^{* *} P<0.001$, and ${ }^{* * * *} P<0.0001($ analysis was by two-way analysis of variance, followed by Fisher's least significant difference multiple-comparison test). 
A

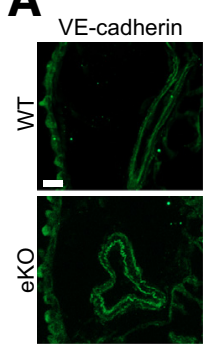

D
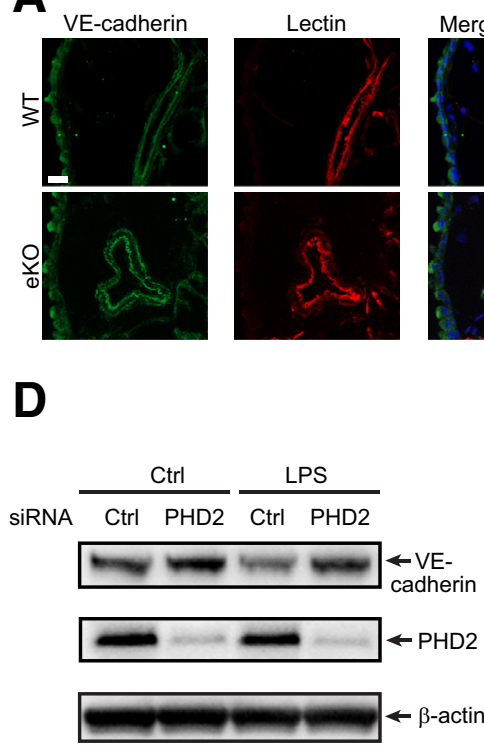

B

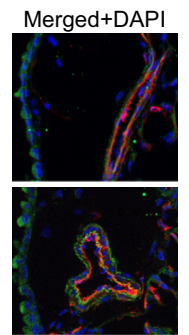

E

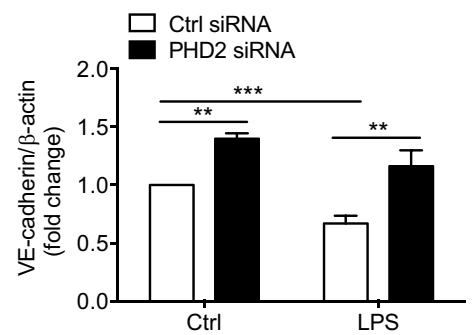

C

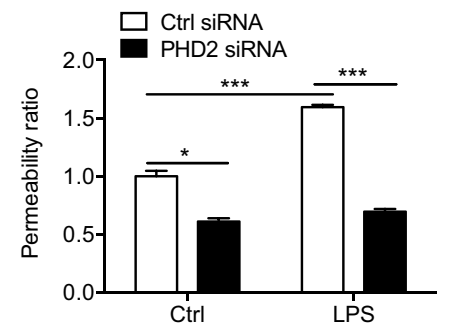

$\mathbf{F}$

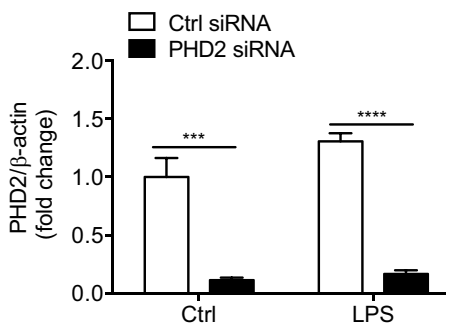

Figure 3 PHD2 deficiency in endothelial cells (ECs) reduces cell permeability by increasing VE-cadherin expression. A and B: Mice with PHD2 depletion in ECs display increased VE-cadherin signals in lung tissue sections compared with WT mice in response to lipopolysaccharide (LPS) treatments. Tissue sections were stained with VE-cadherin antibody (green), lectin (red) for vascular cells, and DAPI (blue) for nucleus. A and B: Representative images are shown (A), and quantified data are presented (B). C: Permeability of mouse lung endothelial cells transfected with control or PHD2 siRNAs was measured after LPS (1 $\mu \mathrm{g} / \mathrm{mL}$ ) or control (Ctrl) treatments. D-F: Microvascular primary endothelial cells transfected with control or PHD2 siRNAs; Western blot analysis was performed to determine protein level changes of VE-cadherin. $\mathbf{E}$ and F: Data of quantitative analysis for Western blot analysis are shown for VE-cadherin (E) and PHD2 (F). Data are expressed as means \pm SEM. $n=4$ (A and $\mathbf{B}$, per section, and $\mathbf{C}) ; n=6$ to 7 per group $(\mathbf{A}$ and $\mathbf{B}) ; n=5(\mathbf{D}-\mathbf{F}) .{ }^{*} P<0.05, * * P<0.01$, $* * * P<0.001$, and $* * * * P<0.0001$ [analysis was by unpaired $t$-test $(\mathbf{B})$ and two-way analysis of variance, followed by Fisher's least significant difference multiple-comparison test $(\mathbf{C}, \mathbf{E}$, and $\mathbf{F})]$. Scale bar $=20 \mu \mathrm{m}(\mathbf{A})$. Original magnification, $\times 400(\mathbf{A})$.

of PHD2 depletion were also assessed on endothelial permeability with an in vitro assay in which the increased flux of a macromolecular tracer FITC-dextran across the endothelial monolayer is indicative of the decreased barrier function. Consistent with less extravasated EBD content in the lung tissue and alleviated vascular leakiness observed in PHD2 eKO mice (Figure 1), in mouse lung MLECs, there was 2.2-fold more solute flux of FITC-dextran across the endothelial monolayer, indicating that LPS increases paracellular permeability (Figure 3C). However, this LPSmediated permeability increase was almost completely blocked by PHD2 knockdown. In parallel, the protein level of VE-cadherin was decreased in MLECs on LPS treatment (Figure 3, D-F). However, PHD2 knockdown significantly increased the VE-cadherin protein level at both basal conditions and on LPS treatment (Figure 3, D-F). Increased VE-cadherin protein levels were also observed in control or LPS-treated ECs isolated from PHD2 eKO mice compared with WT cells (Supplemental Figure S2). These data suggest that PHD2 depletion preserves endothelial barrier function through maintaining VE-cadherin abundance and thereby AJ integrity. Given that pulmonary microvascular endothelial cell apoptosis can also contribute to vessel barrier dysfunction and edema during sepsis ${ }^{41}$ it was also investigated whether PHD2 depletion regulates endothelial apoptosis. Not surprisingly, LPS treatment induced endothelial cell apoptosis, as measured by terminal deoxynucleotidyl transferase-mediated dUTP nick-end labeling analysis with MLECs (Supplemental Figure S3). However, terminal deoxynucleotidyl transferase-mediated dUTP nick-end labeling-positive cell number significantly decreased in PHD2 knockdown MLECs. This suggests that PHD2 depletion may also protect pulmonary endothelial barrier function through inhibiting endothelial apoptosis.

PHD2 Deficiency Regulates VE-Cadherin Protein Level via Stabilizing HIFs, Up-Regulating VE-PTP, and Promoting ROS-Dependent p38 Activity

Recent reports show that HIF $2 \alpha$ maintains endothelial barrier integrity through increasing VE-PTP expression, which prevents VE-cadherin endocytosis and, therefore, stabilizes VE-cadherin junctions. ${ }^{19}$ Given HIFs are main targets of PHDs for hydroxylation, which leads to the subsequent degradation of HIFs in proteasome, ${ }^{42}$ it is likely that the stabilized VE-cadherin protein level in PHD2 knockdown MLECs results from HIF protein stabilization. To further understand the underlying molecular mechanism by which PHD2 depletion induces VE-cadherin protein level in response to LPS stimulation, the stabilization of HIFs in response to LPS and PHD2 depletion was first evaluated. HIF1 $\alpha$ and HIF2 $\alpha$ protein levels remained minimal, whereas PHD2 protein levels were similarly high in both control and LPS-treated ECs (Figure 4, A-C, and 

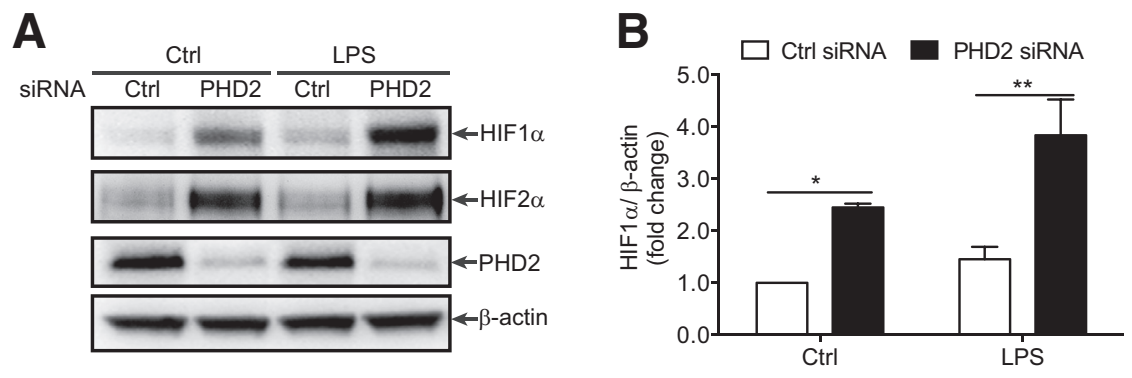

C

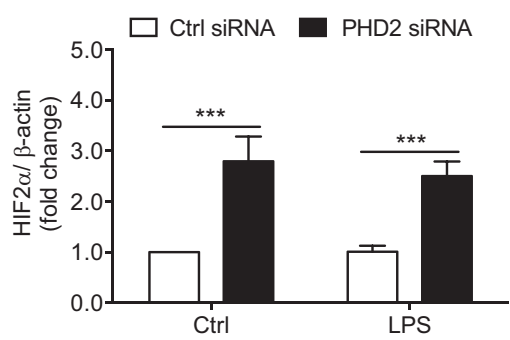

D

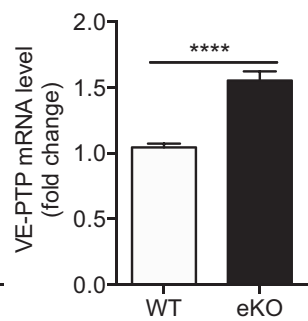

E

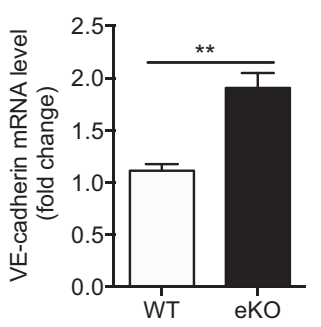

Figure 4 PHD2 regulates the stabilization of HIF1 and HIF2 and the up-regulation of VE-PTP and VE-cadherin in endothelial cells. A-C: Microvascular primary endothelial cells (MLECs) were transfected with PHD2 siRNAs or control (Ctrl) siRNAs. Western blot analysis was performed, as indicated. D and E: MLECs were isolated from PHD2 eKO or WT mice. cDNAs were prepared from total mRNA of indicated cells. Real-time PCR assays for VE-PTP and VE-cadherin were performed. Data are expressed as means \pm SEM. $n=3$ (B, HIF1 $\alpha) ; n=6$ (C, HIF2 $\alpha) ; n=3$ (D and E). ${ }^{*} P<0.05,{ }^{* *} P<0.01,{ }^{* * *} P<0.001$, and $* * * * P<0.0001$ [analysis was by two-way analysis of variance, followed by Fisher's least significant difference multiple-comparison test (B and $\mathbf{C}$ ) and unpaired $t$-test (D and E)]. LPS, lipopolysaccharide.
Supplemental Figure S2), suggesting that basal PHD activity is sufficient to degrade HIF protein. However, PHD2 depletion stabilized both HIF1 $\alpha$ and HIF2 $\alpha$ at basal conditions and in response to LPS treatment in MLECs (Figure 4, A-C). Consistent with the increased protein levels of HIFs, VE-PTP mRNA level was increased in PHD2-depleted ECs (Figure 4D). This further supports that VE-cadherin protein can be stabilized via the PHDHIF-VE-PTP pathway. Surprisingly, VE-cadherin was also up-regulated at the RNA level in PHD2-depleted ECs (Figure 4E). This suggests that PHD2 may regulate VEcadherin abundance through its stability but also gene expression. However, VEGF receptor 2 phosphorylation on VEGF stimulation was not affected by PHD2 knockdown (Supplemental Figure S4), suggesting that VEGF signaling might not be involved in PHD2-dependent regulation of endothelial barrier function.

To further understand the signaling pathways that are responsible for PHD2 depletion-induced VE-cadherin protein induction, multiple signaling pathways were screened. Among these pathways, p38 activity was significantly increased up to approximately 2.3-fold in PHD2 knockdown MLECs (Figure 5, A and B). In the presence of LPS, PHD2 deletion sustained the increase of p38 phosphorylation (Supplemental Figure S5, A and B). It is well documented that ROS signaling plays a pivotal role in mediating p38 activation. ${ }^{43}$ To learn how PHD2 inhibition results in p38 activation, the influence of PHD2 on the generation of ROS was investigated. The fluorescent dyes CM- $\mathrm{H}_{2}$ DCFDA and DHE were used to monitor the production of hydrogen peroxide and superoxide, respectively. When MLECs were transfected with PHD2 siRNAs, an increase in fluorescent DCFDA signal was detected, indicating the increased hydrogen peroxide production
(Figure 5C). Similarly, PHD2 knockdown also led to an increased DHE signal (Figure 5D). In response to LPS, PHD2 depletion further increased DCFDA and DHE signals (Supplemental Figure S5, C and D). In addition, the expression and translocation of NRF-2, a key endogenous sensor of oxidative stress, were measured. These data demonstrate that PHD2 depletion or LPS treatment did not alter the expression level of NRF-2 (Supplemental Figure S5E). Notably, LPS increased the nuclear localization of NRF-2, demonstrating an increased nuclear/cytoplasmic ratio of NRF-2 signals (Supplemental Figure S5, F and $\mathrm{G})$. In addition, PHD2 depletion further promoted the nuclear translocation of NRF-2. Taken together, this suggests that PHD2 deficiency promotes the ROS production at both basal conditions and in response to LPS. Then, it was tested whether ROS generation is required for p38 activation on PHD2 depletion in MLECs. As expected, NAC, an antioxidant reagent, significantly inhibited p38 phosphorylation induced by PHD2 depletion at basal conditions and in response to LPS (Figure 5, E and F, and Supplemental Figure S5, A and B). This suggests that PHD2 depletion results in p38 activation via ROS signaling.

Given that $\mathrm{p} 38$ activity regulates the expression and assembly/disassembly dynamics of VE-cadherin junction, ${ }^{44,45}$ it was evaluated whether ROS-dependent p38 signaling is required for the increase of VE-cadherin protein level on PHD2 depletion. First, ROS generation was blocked in MLECs with NAC and then it was determined whether VEcadherin induction in PHD2-depleted MLECs could be inhibited by NAC treatments. Indeed, PHD2 knockdown by its specific siRNAs significantly increased VE-cadherin protein levels at basal conditions and in response to LPS treatment, compared with MLECs transfected with control siRNAs (Figure 6, A and B). NAC, compared with control 
A

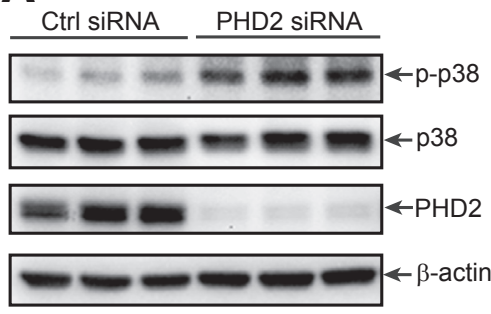

E

siRNA Ctrl PHD2 $\frac{\text { Ctrl }}{\text { Ctrl PHD2 }}$

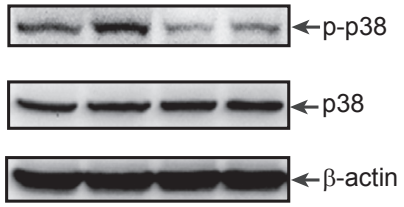

B

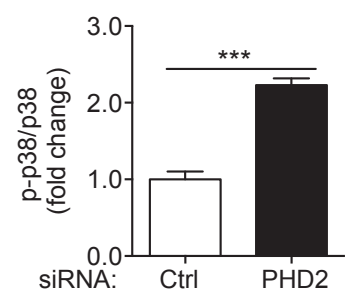

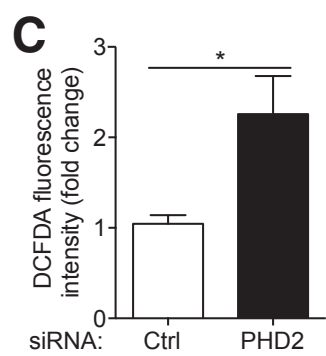

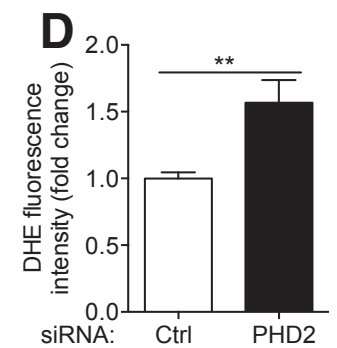

Figure 5 PHD2 depletion increases p38 activity through reactive oxygen species (ROS) generation in endothelial cells. Microvascular primary endothelial cells (MLECs) were transfected with PHD2 or control (Ctrl) siRNAs. A and B: p38 Activation was evaluated as the phosphorylation (p-) of p38 by Western blot analysis. C and D: ROS, including hydrogen peroxide and superoxide, were detected as green fluorescence after $\mathrm{CM}-\mathrm{H}_{2}$ DCFDA or dihydroethidium (DHE) staining. Signals were presented as fold changes compared with control cells treated with control siRNA. $\mathbf{E}$ and $\mathbf{F}$ : MLECs were transfected with PHD2 or control siRNAs. Two days later, they were treated with $10 \mathrm{mmol} / \mathrm{L} \mathrm{N}$-acetyl- L-cysteine (NAC) for 1 hour and then harvested for Western blot analysis. Data are expressed as means \pm SEM. $n=3$ (B and C); $n=6$ (D); $n=5$ (F). ${ }^{*} P<0.05,{ }^{* *} P<0.01$, and ${ }^{* * *} P<0.001$ [analysis was by unpaired $t$ test (B-D) and two-way analysis of variance, followed by Fisher's least significant difference multiple-comparison test $(\mathbf{F})]$.

treatments, prevented the increases of VE-cadherin protein levels in PHD2-depleted MLECs at basal conditions and in response to LPS (Figure 6, A and B). In addition, DPI, a potent inhibitor of NADPH oxidase-dependent ROS production, was used to test whether NADPH oxidase-generated ROS are mediators for PHD2-associated VE-cadherin expression. The data demonstrate that DPI treatment blocked PHD2 deletion-induced VE-cadherin expression at basal conditions and on LPS treatment (Supplemental Figure S6). Next, it was tested if p38 inhibition blocks PHD2 depletion-induced VE-cadherin protein. Similarly to antioxidants NAC and DPI, p38 inhibition by its specific inhibitor SB203580 or its specific siRNAs blocked increases of VE-cadherin protein levels in response to PHD2 knockdown at basal conditions and in response to LPS treatment (Figure 6, C and D, and Supplemental Figure S6). Therefore, the experiments with the antioxidants NAC and DPI and p38 inhibitor or siRNAs indicate that the NADPH oxidase-dependent ROS-p38 signaling is required for PHD2 depletion-induced VE-cadherin in endothelial cells.

PHD2 depletion increases both HIF and ROS-p38 signaling. Recent studies show that HIF $2 \alpha$ increases VEPTP expression and VE-cadherin stability and thereby enhances the integrity of endothelial adherens junctions. ${ }^{19}$ HIF $2 \alpha$ depletion by its siRNAs disrupts the hypoxiainduced stabilization of the endothelial barrier function. ${ }^{19}$ To further determine the involvement of p38 activity and ROS in the regulation of barrier protective function of PHD2 depletion, additional permeability assays were performed with p38 inhibitor SB203580 or antioxidant NAC. The data show that PHD2 depletion decreased EC permeability, indicating its protective effects on endothelial barrier integrity. The inhibition of p38 with SB203580 or antioxidant NAC relieved the inhibitory effects of PHD2 depletion on EC permeability at basal conditions and in response to LPS (Supplemental Figure S7, A and B). It is notable that NAC treatment also inhibited the induced permeability effect of LPS in control cells, suggesting that ROS are also required for LPS-induced permeability. The differential roles of ROS in LPS and PHD2 signaling remain to be further investigated. Given that both the decrease of EC apoptosis and the increase of VE-cadherin expression can inhibit EC permeability, it was also tested whether ROS/p38 signaling is required for the protective effects of PHD2 depletion on LPS-induced apoptosis. The data demonstrate that NAC, but not SB203580, blocked the antiapoptotic effect of PHD2 depletion (Supplemental Figure S8, A and B). This suggests that p38 signaling is required for the protective effects of PHD2 depletion on VE-cadherin expression and the integrity of adherens junction. However, PHD2 depletion protects ECs from apoptosis through a different mechanism that remains to be further characterized.

Taken all together, these data indicate that endothelial PHD2 plays a pivotal role in vascular inflammatory responses during LPS-induced endotoxemia. Its action is mediated through multiple endothelial cell processes, including the production of proinflammatory cytokines, apoptosis, and regulated endothelial barrier function. Further biochemical studies suggest that HIF-VE-PTP and p38 pathways are involved in PHD2-regulated VE-cadherin abundance and endothelial AJ integrity, which eventually 
A

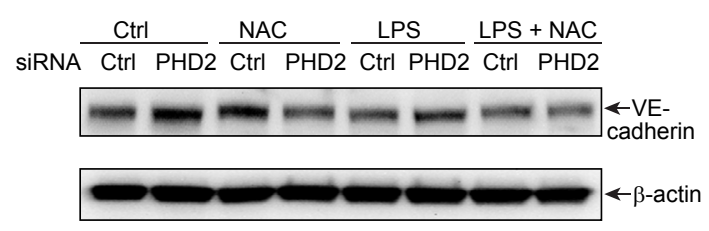

C

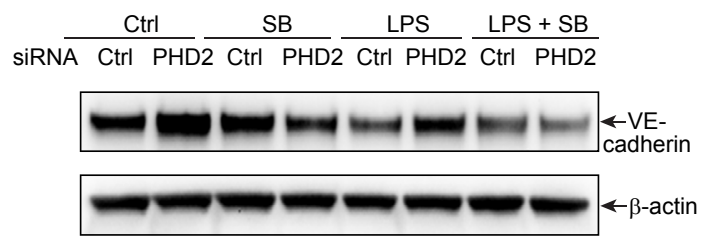

B

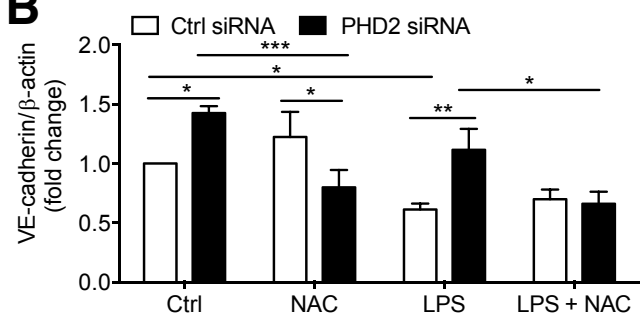

D

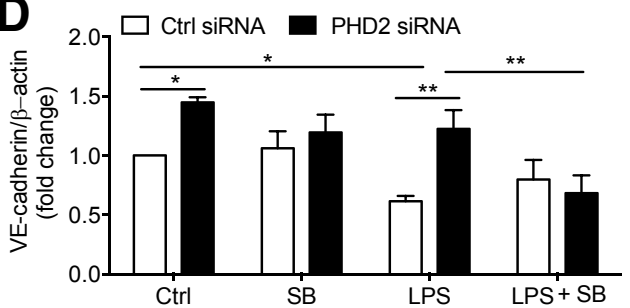

$\mathbf{E}$

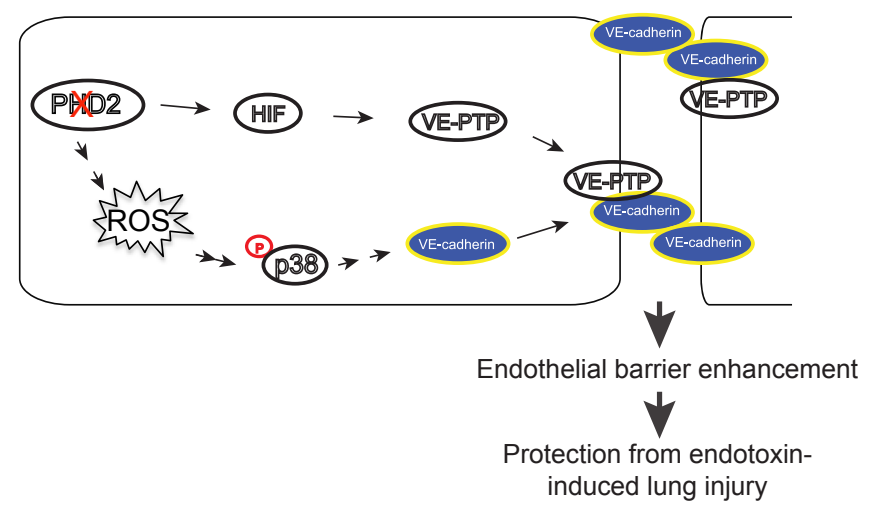

Figure 6 PHD2 depletion increases VE-cadherin level through reactive oxygen species generation and p38 activation in endothelial cells. A-D: Microvascular primary endothelial cells were transfected with PHD2 or control (Ctrl) siRNAs. Two days later, cells were pretreated with $10 \mathrm{mmol} / \mathrm{L} \mathrm{N}$-acetyl-L-cysteine (NAC) or $10 \mu \mathrm{mol} / \mathrm{L} \mathrm{SB203580} \mathrm{(SB)} \mathrm{for} 30$ minutes and then treated with $1 \mu \mathrm{g} / \mathrm{mL}$ lipopolysaccharide (LPS) for 16 hours. Cells were then harvested for Western blot analysis to determine the protein level of VE-cadherin. SB was used to specifically block p38 activity. E: A sketch model to show how PHD2 depletion protects adherens junction integrity and endothelial barrier function. Data are expressed as means \pm SEM. $n=4(\mathbf{B}) ; n=5(\mathbf{D}) .{ }^{*} P<0.05,{ }^{* *} P<0.01$, and $* * * P<0.001$ (analysis was by two-way analysis of variance, followed by Fisher's least significant difference multiple-comparison test).

leads to the changes in endothelial permeability (Figure 6E). These findings provide new insights for PHD2 as a potential therapeutic target for acute lung injury and related inflammatory diseases.

\section{Discussion}

Using endothelial cell-specific inducible knockout mouse models, we demonstrate that endothelial depletion of PHD2 protects mice from LPS-induced overwhelming inflammation and death. In vivo evidence from this study indicates that alleviated injury of PHD2 eKO mice from endotoxemia was mainly due to the improvement of endothelial barrier function. Specifically, decreased lethality of PHD2 eKO mice occurred concomitantly with decreased pulmonary edema and capillary permeability, indicated by lung wet/dry weight ratio and extravasated EBD contents. Leukocyte transmigration and the secretion of inflammatory cytokine into BALF were blocked in PHD2 eKO mice. In addition, the LPS-induced defective AJ junction was stabilized in PHD2-deficient endothelial cells, indicated by increased VE-cadherin intensity on the endothelial cell membrane. This study provides direct in vivo evidence to suggest that endothelial PHD2 plays a pivotal role in LPS-induced vascular inflammatory responses.

Consistently, a series of in vitro functional assays demonstrate that PHD2 knockdown results in a dramatically improved barrier function of lung microvascular endothelial cells. For example, PHD2 knockdown decreased paracellular permeability of FITC-dextran across an MLEC cell layer on LPS stimulation. Further experiments demonstrate that VE-cadherin is induced and stabilized in PHD2-depleted endothelial cells. In addition, endothelial apoptosis is inhibited by PHD2 deficiency. All these lines of evidences indicate that endothelial PHD2 activity controls barrier function through regulating endothelial AJ integrity and cell survival responses. The mechanistic studies suggest that VE-cadherin is a main target of PHD2 activity for the maintenance of $\mathrm{AJ}$ function. However, it is unclear about 
how PHD2 regulates endothelial cell apoptosis. PHD2 inhibition can block apoptosis of kidney epithelial cells and cancer cells through stabilizing HIF and regulatory B subunit of protein phosphatase 2 pathways. ${ }^{46,47}$ It will be interesting to further determine their roles in PHD2regulated endothelial apoptotic responses and $\mathrm{AJ}$ integrity.

HIF $2 \alpha$ eKO mice, generated by the breeding of HIF- $2 \alpha^{\mathrm{f} / \mathrm{f}}$ and $\mathrm{Tie} 2 \mathrm{Cre}{ }^{+/-}$mice, demonstrate aggravated LPS-induced death events and defective endothelial barrier integrity. This proinflammatory phenotype of HIF2 eKO mice is opposite to the anti-inflammatory one for our PHD2-inducible eKO mice, suggesting that PHD2 deficiency might regulate endothelial barrier function through increased HIF2 $\alpha$ activity. HIF $2 \alpha$ increases the expression of VE-PTP and stabilizing VE-cadherin junctions. ${ }^{19}$ Kobayashi et al ${ }^{48}$ have performed chromatin immunoprecipitation assays and demonstrated that both HIF $1 \alpha$ and HIF $2 \alpha$ are associated with the potential hypoxia-responsive element of the VEcadherin's promoter in ECs. Because the in vitro studies confirm that PHD2 depletion increased the protein levels of HIF1 $\alpha$, HIF2 $\alpha$, VE-PTP, and VE-cadherin, it suggests that PHD2 depletion restores endothelial barrier function through improving the HIF $1 \alpha$, HIF $2 \alpha$, VE-PTP, and VEcadherin signaling cascades and $\mathrm{AJ}$ integrity. In addition, PHD2 depletion results in other changes, including the ROS-p38 signaling-dependent induction of VE-cadherin expression. Therefore, PHD2 depletion can regulate VE-cadherin abundance through two mechanisms: its stabilization via the HIF-dependent pathway and its expression via both HIF and ROS-p38 pathways. It also suggests that the protective phenotype in PHD2 eKO mice is likely mediated through both HIF-dependent and HIFindependent pathways. Hypoxia induces ROS, and PHD1 inhibition reduces ROS production in ischemic myofibers. ${ }^{25}$ Interestingly, PHD2 inhibition increased ROS generation in endothelial cells, which is required for p38 activation and subsequent VE-cadherin protein induction. It is worthwhile to speculate that PHD2 regulates NADPH oxidases or other enzyme- or non-enzyme-dependent systems responsible for ROS generation, which needs to be studied further. Previous reports demonstrate that VEGF receptor 2 and VEGF were mildly up-regulated in PHD2-depleted retinas. ${ }^{49}$ However, little retinal neoangiogenesis was detected in PHD2inducible $\mathrm{KO}$ mice. ${ }^{49}$ Interestingly, increases of VEGF receptor 2 expression and phosphorylation were not observed in PHD2-depleted MLECs, although HIF1 $\alpha$ and HIF2 $\alpha$ were stabilized (Figure 4, A-C, and Supplemental Figure S4). This suggests that VEGF signaling is not required for the regulation of endothelial barrier function by the PHD2-HIF signaling axis. In addition, PHD2 depletion in ECs of different vessel beds may have variable impacts on the VEGF pathway. Combinational inhibition of other PHDs might be also required for the full response of the VEGF pathway.

Recent data have demonstrated that PHDs may regulate cellular processes through HIF-dependent and
HIF-independent pathways, including the large subunit of RNA polymerase, $\beta 2$-adrenergic receptor, pyruvate kinase M2, and caenorhabditis elegans biological clock protein CLK-2. ${ }^{50-54}$ Recent studies show that endothelial depletion of PHD2 increases pulmonary vascular remodeling through promoting pericyte coverage and perivascular interstitial fibrosis. ${ }^{55} \mathrm{~A}$ list of HIF target genes are induced in PHD2depleted mouse lung tissue, including fibroblast-specific protein-1, Notch2, and transforming growth factor- $\beta .^{56}$ These proteins might also contribute to the protective effects of PHD2 depletion on endothelial permeability, apoptosis, or inflammatory response during lung injury. Moreover, PHDs have been suggested to hydroxylate the I $\kappa \mathrm{B}$ kinase and, therefore, repress NF- $\kappa \mathrm{B}$ activity. ${ }^{50,51} \mathrm{Re}-$ ports also show that PHD3 regulates the NF- $\kappa \mathrm{B}$ pathway through inhibiting IKB kinase $\gamma$ ubiquitination. ${ }^{57}$ It is reasonable to hypothesize that PHD2 may also regulate septic inflammation through regulating the NF- $\kappa B$ pathway. However, when PHD2 is depleted in endothelial cells, no effects have been observed with $\mathrm{I} \kappa \mathrm{B}$ kinase activity, I $\mathrm{B}$ degradation, and induction of NF- $\kappa \mathrm{B}$ target genes [VCAMI and ICAMI (data not shown)]. This suggests that the NF- $\kappa \mathrm{B}$ pathway, a main target of PHD3 signaling, does not contribute significantly to PHD2-dependent regulation in inflammatory responses during LPS-induced endotoxemia.

Significant progress has been made for testing the protective effects of hydroxylase inhibition in the treatment of complex inflammatory disorders, such as sepsis, inflammatory bowel disease, hepatic and intestinal disorders, and tumorigenesis. ${ }^{58,59}$ Initial studies have demonstrated that hydroxylase inhibitors DMOG and FG-4497 can decrease inflammatory responses in dextran-sodium sulfate-induced colitis. ${ }^{60,61}$ The pretreatment with DMOG suppresses LPSinduced TNF- $\alpha$ expression and alleviates endotoxic shock (Figure 1C). ${ }^{32,62}$ PHD2 inhibition by its inhibitor FG4497 prevents LPS- and cecal ligation and puncture-induced lung injury. Recent studies also characterized the roles of PHDs in systemic inflammation and sepsis with their globe knockout models. PHD3 global deficiency aggravates abdominal sepsis, likely via hyperactivated innate immune cells, whereas PHD1 deficiency or PHD2 haploinsufficiency has no or mild effects. ${ }^{30}$ The data with PHD2/3 eKO mice demonstrate that the depletion of both PHD2 and PHD3, compared with PHD2 eKO mice, similarly protects mice from LPS-induced death. They indicate that inhibition of PHD2 activity in endothelial cells is sufficient to block detrimental effects of LPS-induced endotoxemia. In addition, PHD3 eKO mice displayed a low survival rate (Supplemental Figure S1B). Therefore, we speculate that $\mathrm{PHD}^{-1-}$ innate immune cells, ${ }^{30}$ but not endothelial cells, play a main role in detrimental effects of PHD3 global depletion during sepsis shock. Taken together, these data support that individual PHD isoforms in different cell types possess specific and nonredundant in vivo functions. All these studies raise an urgent need for the development of PHD isoform-specific inhibitors that are expected to 
improve the efficacy and safety of the prolyl hydroxylase inhibition in the potential clinical applications.

Hypoxia and inflammation share an interdependent relationship. In an ARDS patient, mortality is associated with an increased degree of hypoxia. Both PHD2 and PHD3 are increased by hypoxia in a variety of human tissues. ${ }^{63}$ In human neutrophils, PHD3 is strongly induced in response to hypoxia and inflammatory stimuli in vitro and in vivo. ${ }^{64}$ Thus, the inhibition of PHD may become a potential strategy for the treatment of inflammatory diseases, such as ARDS. However, only approximately $10 \%$ to $15 \%$ of patients die of refractory hypoxemia. This might be because of the protective role of endothelial PHD2 inhibition, which counterbalances the hyperactivated innate immune responses induced by inhibited PHD3 activity in macrophages. Therefore, specific inhibitors designed against PHD2 may become a new and safe therapeutic option to improve the endothelial barrier-protective mechanism in ARDS patients.

\section{Acknowledgments}

We thank the Baylor College of Medicine Histology Core and Optical Imaging and Vital Microscopy Core for help; and Dr. Ralf H. Adams for providing Cdh5-CreER ${ }^{+l-}$ transgenic mice.

Q.F., L.X., and X.P. conceived the research and designed the experiments; Q.F., H.M., and X.P. performed the experiments; Q.F., L.X., and X.P. wrote the manuscript; and all authors discussed the results and commented on the manuscript.

\section{Supplemental Data}

Supplemental material for this article can be found at https://doi.org/10.1016/j.ajpath.2018.09.012.

\section{References}

1. Ragaller M, Richter T: Acute lung injury and acute respiratory distress syndrome. J Emerg Trauma Shock 2010, 3:43-51

2. Johnson ER, Matthay MA: Acute lung injury: epidemiology, pathogenesis, and treatment. J Aerosol Med Pulm Drug Deliv 2010, 23: $243-252$

3. Maniatis NA, Kotanidou A, Catravas JD, Orfanos SE: Endothelial pathomechanisms in acute lung injury. Vascul Pharmacol 2008, 49: 119-133

4. Mehta D, Malik AB: Signaling mechanisms regulating endothelial permeability. Physiol Rev 2006, 86:279-367

5. Orfanos SE, Mavrommati I, Korovesi I, Roussos C: Pulmonary endothelium in acute lung injury: from basic science to the critically ill. Intensive Care Med 2004, 30:1702-1714

6. Sukriti S, Tauseef M, Yazbeck P, Mehta D: Mechanisms regulating endothelial permeability. Pulm Circ 2014, 4:535-551

7. Dejana E, Giampietro C: Vascular endothelial-cadherin and vascular stability. Curr Opin Hematol 2012, 19:218-223

8. Runkle EA, Mu D: Tight junction proteins: from barrier to tumorigenesis. Cancer Lett 2013, 337:41-48
9. Lakshmi SP, Reddy AT, Naik MU, Naik UP, Reddy RC: Effects of JAM-A deficiency or blocking antibodies on neutrophil migration and lung injury in a murine model of ALI. Am J Physiol Lung Cell Mol Physiol 2012, 303:L758-L766

10. Goodenough DA, Paul DL: Beyond the gap: functions of unpaired connexon channels. Nat Rev Mol Cell Biol 2003, 4:285-294

11. Vandenbroucke St Amant E, Tauseef M, Vogel SM, Gao XP, Mehta D, Komarova YA, Malik AB: PKCalpha activation of p120catenin serine 879 phospho-switch disassembles VE-cadherin junctions and disrupts vascular integrity. Circ Res 2012, 111: 739-749

12. Potter MD, Barbero S, Cheresh DA: Tyrosine phosphorylation of VEcadherin prevents binding of p120- and beta-catenin and maintains the cellular mesenchymal state. J Biol Chem 2005, 280: 31906-31912

13. Nottebaum AF, Cagna G, Winderlich M, Gamp AC, Linnepe R, Polaschegg C, Filippova K, Lyck R, Engelhardt B, Kamenyeva O, Bixel MG, Butz S, Vestweber D: VE-PTP maintains the endothelial barrier via plakoglobin and becomes dissociated from VE-cadherin by leukocytes and by VEGF. J Exp Med 2008, 205:2929-2945

14. Broermann A, Winderlich $M$, Block $H$, Frye $M$, Rossaint J, Zarbock A, Cagna G, Linnepe R, Schulte D, Nottebaum AF, Vestweber D: Dissociation of VE-PTP from VE-cadherin is required for leukocyte extravasation and for VEGF-induced vascular permeability in vivo. J Exp Med 2011, 208:2393-2401

15. Le Bras A, Lionneton F, Mattot V, Lelievre E, Caetano B, Spruyt N, Soncin F: HIF-2alpha specifically activates the VE-cadherin promoter independently of hypoxia and in synergy with Ets-1 through two essential ETS-binding sites. Oncogene 2007, 26:7480-7489

16. Birdsey GM, Dryden NH, Amsellem V, Gebhardt F, Sahnan K, Haskard DO, Dejana E, Mason JC, Randi AM: Transcription factor Erg regulates angiogenesis and endothelial apoptosis through VEcadherin. Blood 2008, 111:3498-3506

17. Deleuze V, Chalhoub E, El-Hajj R, Dohet C, Le Clech M, Couraud PO, Huber P, Mathieu D: TAL-1/SCL and its partners E47 and LMO2 up-regulate VE-cadherin expression in endothelial cells. Mol Cell Biol 2007, 27:2687-2697

18. Eltzschig HK, Carmeliet P: Hypoxia and inflammation. N Engl J Med 2011, 364:656-665

19. Gong H, Rehman J, Tang H, Wary K, Mittal M, Chaturvedi P, Zhao YY, Komarova YA, Vogel SM, Malik AB: HIF2alpha signaling inhibits adherens junctional disruption in acute lung injury. J Clin Invest 2015, 125:652-664

20. Bruick RK, McKnight SL: A conserved family of prolyl-4hydroxylases that modify HIF. Science 2001, 294:1337-1340

21. Ivan M, Kondo K, Yang H, Kim W, Valiando J, Ohh M, Salic A, Asara JM, Lane WS, Kaelin WG Jr: HIFalpha targeted for VHLmediated destruction by proline hydroxylation: implications for $\mathrm{O} 2$ sensing. Science 2001, 292:464-468

22. Jaakkola P, Mole DR, Tian YM, Wilson MI, Gielbert J, Gaskell SJ, von Kriegsheim A, Hebestreit HF, Mukherji M, Schofield CJ, Maxwell PH, Pugh CW, Ratcliffe PJ: Targeting of HIF-alpha to the von Hippel-Lindau ubiquitylation complex by O2-regulated prolyl hydroxylation. Science 2001, 292:468-472

23. Fong GH, Takeda K: Role and regulation of prolyl hydroxylase domain proteins. Cell Death Differ 2008, 15:635-641

24. Schneider M, Van Geyte K, Fraisl P, Kiss J, Aragones J, Mazzone M, Mairbaurl H, De Bock K, Jeoung NH, Mollenhauer M, Georgiadou M, Bishop T, Roncal C, Sutherland A, Jordan B, Gallez B, Weitz J, Harris RA, Maxwell P, Baes M, Ratcliffe P, Carmeliet P: Loss or silencing of the PHD1 prolyl hydroxylase protects livers of mice against ischemia/reperfusion injury. Gastroenterology 2010, 138:1143-1154.e1-2

25. Aragones J, Schneider M, Van Geyte K, Fraisl P, Dresselaers T, Mazzone M, et al: Deficiency or inhibition of oxygen sensor Phd1 induces hypoxia tolerance by reprogramming basal metabolism. Nat Genet 2008, 40:170-180 
26. Minamishima YA, Moslehi J, Bardeesy N, Cullen D, Bronson RT, Kaelin WG Jr: Somatic inactivation of the PHD2 prolyl hydroxylase causes polycythemia and congestive heart failure. Blood 2008, 111: 3236-3244

27. Bishop T, Gallagher D, Pascual A, Lygate CA, de Bono JP, Nicholls LG, Ortega-Saenz P, Oster H, Wijeyekoon B, Sutherland AI, Grosfeld A, Aragones J, Schneider M, van Geyte K, Teixeira D, Diez-Juan A, Lopez-Barneo J, Channon KM, Maxwell PH, Pugh CW, Davies AM, Carmeliet P, Ratcliffe PJ: Abnormal sympathoadrenal development and systemic hypotension in PHD3-/mice. Mol Cell Biol 2008, 28:3386-3400

28. Minamishima YA, Moslehi J, Padera RF, Bronson RT, Liao R, Kaelin WG Jr: A feedback loop involving the Phd3 prolyl hydroxylase tunes the mammalian hypoxic response in vivo. Mol Cell Biol 2009, 29:5729-5741

29. Xie L, Pi X, Townley-Tilson WH, Li N, Wehrens XH, Entman ML, Taffet GE, Mishra A, Peng J, Schisler JC, Meissner G, Patterson C: PHD2/3-dependent hydroxylation tunes cardiac response to betaadrenergic stress via phospholamban. J Clin Invest 2015, 125: 2759-2771

30. Kiss J, Mollenhauer M, Walmsley SR, Kirchberg J, Radhakrishnan P, Niemietz T, Dudda J, Steinert G, Whyte MK, Carmeliet P, Mazzone M, Weitz J, Schneider M: Loss of the oxygen sensor PHD3 enhances the innate immune response to abdominal sepsis. J Immunol 2012, 189:1955-1965

31. Tambuwala MM, Cummins EP, Lenihan CR, Kiss J, Stauch M, Scholz CC, Fraisl P, Lasitschka F, Mollenhauer M, Saunders SP, Maxwell PH, Carmeliet P, Fallon PG, Schneider M, Taylor CT: Loss of prolyl hydroxylase-1 protects against colitis through reduced epithelial cell apoptosis and increased barrier function. Gastroenterology 2010, 139:2093-2101

32. Takeda $\mathrm{K}$, Ichiki $\mathrm{T}$, Narabayashi $\mathrm{E}$, Inanaga $\mathrm{K}$, Miyazaki $\mathrm{R}$, Hashimoto T, Matsuura H, Ikeda J, Miyata T, Sunagawa K: Inhibition of prolyl hydroxylase domain-containing protein suppressed lipopolysaccharide-induced TNF-alpha expression. Arterioscler Thromb Vasc Biol 2009, 29:2132-2137

33. Lim YC, Garcia-Cardena G, Allport JR, Zervoglos M, Connolly AJ, Gimbrone MA Jr, Luscinskas FW: Heterogeneity of endothelial cells from different organ sites in T-cell subset recruitment. Am J Pathol 2003, 162:1591-1601

34. Pi $\mathrm{X}$, Yan $\mathrm{C}$, Berk BC: Big mitogen-activated protein kinase (BMK1)/ERK5 protects endothelial cells from apoptosis. Circ Res 2004, 94:362-369

35. Mao H, Lockyer P, Townley-Tilson WH, Xie L, Pi X: LRP1 regulates retinal angiogenesis by inhibiting PARP-1 activity and endothelial cell proliferation. Arterioscler Thromb Vasc Biol 2016, 36: 350-360

36. Lockyer P, Mao H, Fan Q, Li L, Yu-Lee LY, Eissa NT, Patterson C, Xie L, Pi X: LRP1-dependent BMPER signaling regulates lipopolysaccharide-induced vascular inflammation. Arterioscler Thromb Vasc Biol 2017, 37:1524-1535

37. Matute-Bello G, Winn RK, Jonas M, Chi EY, Martin TR, Liles WC: Fas (CD95) induces alveolar epithelial cell apoptosis in vivo: implications for acute pulmonary inflammation. Am J Pathol 2001, 158: $153-161$

38. Xie L, Pi X, Wang Z, He J, Willis MS, Patterson C: Depletion of PHD3 protects heart from ischemia/reperfusion injury by inhibiting cardiomyocyte apoptosis. J Mol Cell Cardiol 2015, 80: $156-165$

39. Takeda K, Ho VC, Takeda H, Duan LJ, Nagy A, Fong GH: Placental but not heart defects are associated with elevated hypoxia-inducible factor alpha levels in mice lacking prolyl hydroxylase domain protein 2. Mol Cell Biol 2006, 26:8336-8346

40. Komarova Y, Malik AB: Regulation of endothelial permeability via paracellular and transcellular transport pathways. Annu Rev Physiol 2010, 72:463-493
41. Gill SE, Taneja R, Rohan M, Wang L, Mehta S: Pulmonary microvascular albumin leak is associated with endothelial cell death in murine sepsis-induced lung injury in vivo. PLoS One 2014, 9:e88501

42. Kaelin WG Jr, Ratcliffe PJ: Oxygen sensing by metazoans: the central role of the HIF hydroxylase pathway. Mol Cell 2008, 30:393-402

43. Son Y, Kim S, Chung HT, Pae HO: Reactive oxygen species in the activation of MAP kinases. Methods Enzymol 2013, 528:27-48

44. Nwariaku FE, Chang J, Zhu X, Liu Z, Duffy SL, Halaihel NH, Terada L, Turnage RH: The role of p38 map kinase in tumor necrosis factor-induced redistribution of vascular endothelial cadherin and increased endothelial permeability. Shock 2002, 18:82-85

45. Khanna P, Yunkunis T, Muddana HS, Peng HH, August A, Dong C: p38 MAP kinase is necessary for melanoma-mediated regulation of VE-cadherin disassembly. Am J Physiol Cell Physiol 2010, 298: C1140-C1150

46. Di Conza G, Trusso Cafarello S, Zheng X, Zhang Q, Mazzone M: PHD2 targeting overcomes breast cancer cell death upon glucose starvation in a PP2A/B55alpha-mediated manner. Cell Rep 2017, 18: 2836-2844

47. Fang Y, Zhang H, Zhong Y, Ding X: Prolyl hydroxylase 2 (PHD2) inhibition protects human renal epithelial cells and mice kidney from hypoxia injury. Oncotarget 2016, 7:54317-54328

48. Kobayashi S, Yamashita T, Ohneda K, Nagano M, Kimura K, Nakai H, Poellinger L, Ohneda O: Hypoxia-inducible factor-3alpha promotes angiogenic activity of pulmonary endothelial cells by repressing the expression of the VE-cadherin gene. Genes Cells 2015, 20:224-241

49. Duan LJ, Takeda K, Fong GH: Prolyl hydroxylase domain protein 2 (PHD2) mediates oxygen-induced retinopathy in neonatal mice. Am J Pathol 2011, 178:1881-1890

50. Cummins EP, Berra E, Comerford KM, Ginouves A, Fitzgerald KT, Seeballuck F, Godson C, Nielsen JE, Moynagh P, Pouyssegur J, Taylor CT: Prolyl hydroxylase-1 negatively regulates IkappaB kinase-beta, giving insight into hypoxia-induced NFkappaB activity. Proc Natl Acad Sci U S A 2006, 103:18154-18159

51. Fu J, Taubman MB: Prolyl hydroxylase EGLN3 regulates skeletal myoblast differentiation through an NF-kappaB-dependent pathway. J Biol Chem 2010, 285:8927-8935

52. Xie L, Pi X, Mishra A, Fong G, Peng J, Patterson C: PHD3-dependent hydroxylation of HCLK2 promotes the DNA damage response. J Clin Invest 2012, 122:2827-2836

53. Xie L, Xiao K, Whalen EJ, Forrester MT, Freeman RS, Fong G Gygi SP, Lefkowitz RJ, Stamler JS: Oxygen-regulated beta(2)adrenergic receptor hydroxylation by EGLN3 and ubiquitylation by pVHL. Sci Signal 2009, 2:ra33

54. Yi Y, Mikhaylova O, Mamedova A, Bastola P, Biesiada J, Alshaikh E, Levin L, Sheridan RM, Meller J, Czyzyk-Krzeska MF: von Hippel-Lindau-dependent patterns of RNA polymerase II hydroxylation in human renal clear cell carcinomas. Clin Cancer Res 2010, 16:5142-5152

55. Wang S, Zeng H, Xie XJ, Tao YK, He X, Roman RJ, Aschner JL, Chen JX: Loss of prolyl hydroxylase domain protein 2 in vascular endothelium increases pericyte coverage and promotes pulmonary arterial remodeling. Oncotarget 2016, 7:58848-58861

56. Dai Z, Li M, Wharton J, Zhu MM, Zhao YY: Prolyl-4 hydroxylase 2 (PHD2) deficiency in endothelial cells and hematopoietic cells induces obliterative vascular remodeling and severe pulmonary arterial hypertension in mice and humans through hypoxia-inducible factor2alpha. Circulation 2016, 133:2447-2458

57. Fu J, Taubman MB: EGLN3 inhibition of NF-kappaB is mediated by prolyl hydroxylase-independent inhibition of IkappaB kinase gamma ubiquitination. Mol Cell Biol 2013, 33:3050-3061

58. Harnoss JM, Strowitzki MJ, Radhakrishnan P, Platzer LK, Harnoss JC, Hank T, Cai J, Ulrich A, Schneider M: Therapeutic inhibition of prolyl hydroxylase domain-containing enzymes in surgery: putative applications and challenges. Hypoxia (Auckl) 2015, 3:1-14 
59. Manresa MC, Taylor CT: Hypoxia inducible factor (HIF) hydroxylases as regulators of intestinal epithelial barrier function. Cell Mol Gastroenterol Hepatol 2017, 3:303-315

60. Cummins EP, Seeballuck F, Keely SJ, Mangan NE, Callanan JJ, Fallon PG, Taylor CT: The hydroxylase inhibitor dimethyloxalylglycine is protective in a murine model of colitis. Gastroenterology 2008, 134:156-165

61. Robinson A, Keely S, Karhausen J, Gerich ME, Furuta GT, Colgan SP: Mucosal protection by hypoxia-inducible factor prolyl hydroxylase inhibition. Gastroenterology 2008, 134:145-155

62. Hams E, Saunders SP, Cummins EP, O'Connor A, Tambuwala MT, Gallagher WM, Byrne A, Campos-Torres A, Moynagh PM, Jobin C, Taylor CT, Fallon PG: The hydroxylase inhibitor dimethyloxallyl glycine attenuates endotoxic shock via alternative activation of macrophages and IL-10 production by B1 cells. Shock 2011, 36: 295-302

63. Appelhoff RJ, Tian YM, Raval RR, Turley H, Harris AL, Pugh CW, Ratcliffe PJ, Gleadle JM: Differential function of the prolyl hydroxylases PHD1, PHD2, and PHD3 in the regulation of hypoxiainducible factor. J Biol Chem 2004, 279:38458-38465

64. Walmsley SR, Chilvers ER, Thompson AA, Vaughan K, Marriott HM, Parker LC, Shaw G, Parmar S, Schneider M, Sabroe I, Dockrell DH, Milo M, Taylor CT, Johnson RS, Pugh CW, Ratcliffe PJ, Maxwell PH, Carmeliet P, Whyte MK: Prolyl hydroxylase 3 (PHD3) is essential for hypoxic regulation of neutrophilic inflammation in humans and mice. J Clin Invest 2011, 121:1053-1063 